\title{
Structure et Fonctionnement des Écosystèmes du Haut-Rhône Français; VIII: Hydrologie de deux stations phréatiques dont l'eau alimente des bras morts*
}

\author{
J.L. Reygrobellet, J. Mathieu, R. Ginet, J. Gibert**
}

\section{SUMMARY}

We have been working since 1975 on phreatic stations providing two old meanders of the French river Rhône with interstitial water. The hydrological characteristics are quite different (see diagrams in the text):

- The one (station 2) is a particular under-flow circulating laterally to the river ("paraécoulement"'), which is closely subordinate with the varying level of the Rhône.

- The other (station 8 ) is the confluence of continental phreatic water proceeding from a Northeastern plateau (the "Dombes") with the hyporheic of the river Ain (eastern tributary of the Rhône). The Rhône has no influence on the characteristics of this second interstitial flow.

The physico-chemical disparity of these stations clearly appeared during two extremely opposite climatic periods; the inferences on resident populations are considered.

\section{INTRODUCTION}

Depuis 1975, notre Groupe de Recherche effectue une étude exhaustive du fleuve Rhône en amont de Lyon (Roux, 1976). Lors d'un travail préliminaire sur le milieu phréatique environnant, nous avions mentionné (Gibert, et al., 1977) la particularité de certaines eaux souterraines régionales, d'alimenter les restes d'anciens bras ou méandres du fleuve, constituant ainsi des étendues d'eau calme, plus ou moins proches du cours actif, et plus ou moins directement liées à lui suivant leur "âge". Ces "Lônes", successions de plusieurs écotones, forment des milieux originaux dont le secteur émergé fait depuis trois ans l'objet d'une recherche approfondie (Juget, et al., 1976, 1979); corrélativement, notre équipe travaille depuis la même époque sur l'écosystème interstitiel qu'elles confinent.

Pendant trois ans, nous avons donc essayé de cerner à la fois les caractéristiques hydrologiques de l'alimentation de ces lônes par de l'eau phréatique, et les phénomènes biologiques qui y sont liés.

Nous avons sélectionné deux stations d'étude:

- L'une (station 2, Gibert, et al., 1977) peut être considérée comme la

* Publication préliminaire à l'analyse faunistique qui figure à la suite de ce texte (IX).

** Biologie Souterraine, Université Claude Bernard LYON I, 43 Bd. du 11 Novembre 1918, 69622, Villeurbanne - France -. 
source de la "Lône des Pêcheurs"; cette dernière tire son eau du sousécoulement du Rhône et forme, rive gauche, sur plus de deux kilomètres, un véritable "affluent phréatique" du fleuve qui conflue avec lui légèrement en amont du barrage de Jons.

- L'autre (station 8, Gibert, et al., 1977), est constituée par le milieu interstitiel particulier de la 'Lône de l'Ile du Méant', localisée, rive droite, à l'emplacement de l'ancienne confluence de la Rivière l'Ain avec le Rhône.

L'objet de cette publication est de définir les biotopes où nous effectuons nos prélèvements de faune depuis 1975, dont les résultats sont exposés à la suite de ce texte (Gibert, et al., in this volume). Pour caractériser chacune des stations, nous utiliserons trois points de comparaison: le mode d'écoulement interstitiel, la composition granulométrique des sédiments, et la physico-chimie des eaux.

\section{MODE D’ÉCOULEMENT DES EAUX INTERSTITIELLES}

\section{A) "Lône des Pêcheurs"' (Station '2"')}

Depuis Dorgelo (1973), l'historique de la formation des lônes est bien connue. La lône des Pêcheurs s'est isolée du cours actif du Rhône vers 1860 . C'est actuellement la lône la mieux connue de notre groupe de travail, car son étude fait l'objet de la convention de recherche $n^{\circ}$ 76-55 du Ministère de l'Environnement et du Cadre de vie. Elle représente un complexe hydrographique voisin de celui du Waldrhein, situé en aval de Strasbourg (Alsace, France) dans la zone de confluence I11-Rhin (Ortcheit, 1975), qui est séparée du cours principal du fleuve depuis une date équivalente (1885).

Cependant, les fluctuations de niveau dûes aux manœuvres du barrage de Jons, en aval de la confluence Lône des Pêcheurs-Rhône, compliquent ici les phénomènes hydrologiques par rapport au système alsacien, créant des courants rythmiques de reflux à l'intérieur de la lône qui rendent mobile la zone où s'affrontent les eaux d'origine phréatique et les eaux fluviales. Malgré ce handicap, Juget, et al. (1978), utilisant la position relative de cette zone d'affrontement comme révélateur du débit phréatique, ont pu démontrer, pour les eaux alimentant la lône, leur totale dépendance par rapport au débit du Rhône lui-même.

Nous sommes donc en présence d'un “para-écoulement"' interstitiel latéral au Rhône, émergeant en station 2 et engendrant un cours d'eau superficiel "qui se trouve barré en aval par son propre émissaire" (Juget, et al, 1979). Le diagramme de la Figure 1 résume l'ensemble de ces données et fournit une représentation schématisée des courants souterrains environnant la station 2 .

\section{B) "Lône de l'île du Méant" (Station "8")}

Cette lône, dont la station 8 est l'une des zones d'alimentation, est la plus récente des lônes de la région (Dorgelo, 1973). Elle est en effet située, sur la rive droite, immédiatement en aval de la confluence Ain-Rhône qui a évolué en 
remontant vers l'amont. A son ancien emplacement se trouve actuellement ce bras, encore assez vif, dont la partie interstitielle est le lieu de convergence du sous-écoulement de l'Ain (il n'en est pas totalement coupé superficiellement) et de la nappe continentale descendant de la Dombes, perpendiculairement au Rhône, par la terrasse de La Valbonne. L'alimentation de cette lône est donc à la fois plus diffuse et plus complexe que celle de la Lône des Pêcheurs qui n'est qu'un sous-écoulement du Rhône vif.

Cette région a été particulièrement bien étudiée par trois offices régionaux: le Bureau de Recherches de Géologie Minière (B.R.G.M.), l'Agence de Bassin Rhône-Méditerranée-Corse, et le Service Régional d'Aménagement des Eaux (S.R.A.E.), que nous tenons à remercier pour les renseignements complets qu'ils nous ont aimablement fournis. La justification de leur intérêt est que la "Plaine de Miribel-La Valbonne" où se trouve notre station 8 est un réservoir d'eau potable d'une telle capacité qu'on envisage d'en faire la future zone de captage de l'agglomération lyonnaise. De nombreux rapports ont été rédigés par ces organismes depuis 1970, tant sur l'hydrologie du système aquifère (B.R.G.M., 1971 a, b; 1973, 1976) que sur la vulnérabilité aux pollutions de la nappe régionale (Agence de Bassin, 1974; Pinchaut, 1975). Récemment, le S.R.A.E. a même réalisé un forage d'essai à $-18 \mathrm{~m}$ (en vue d'irrigation) dans un secteur très proche de l'aire qui nous intéresse.

De ce fait, les sens d'écoulement, les débits moyens de l'aquifère et la vitesse de la nappe sont actuellement bien connus, ainsi que les importantes possibilités de pompage. Les données physico-chimiques moyennes du phréatique profond le sont aussi, et seront intéressantes à comparer avec les résultats obtenus sur l'interstitiel superficiel.

L'ensemble des informations fournies par ces rapports nous a permis d'établir, pour la station 8 également, un bloc-diagramme d'alimentation

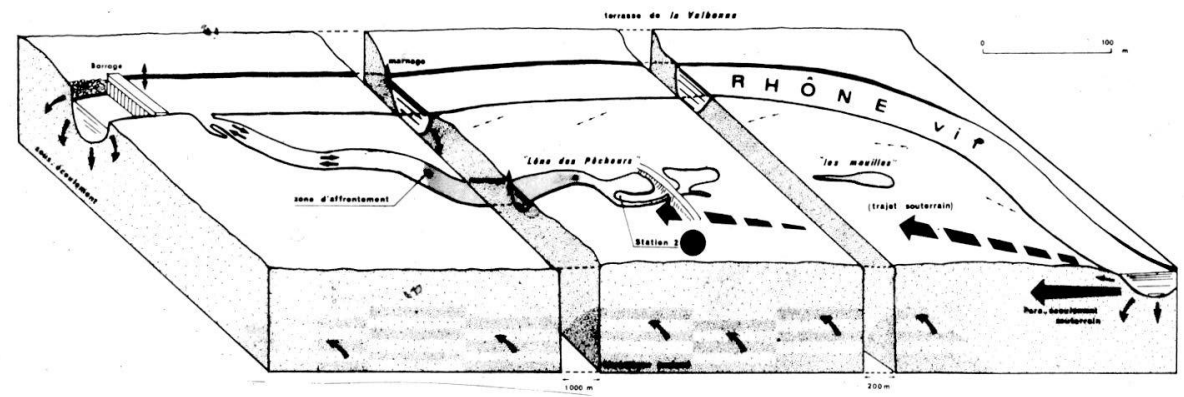

Fig. 1. Courants souterrains dans la zone d'alimentation de la Station 2

$\rightarrow$ : Infiltrations

-.. : courant hyporhéique

: para-écoulement principal 
(Fig. 2): c'est essentiellement, sur tout le cours amont de la Lône de l'ìle du Méant, un rééquilibrage du niveau piézométrique par les multiples remontées d'eau souterraine; ce n'est en effet qu'en périodes de hautes eaux que la lône est soumise aux courants des eaux superficielles de l'Ain.

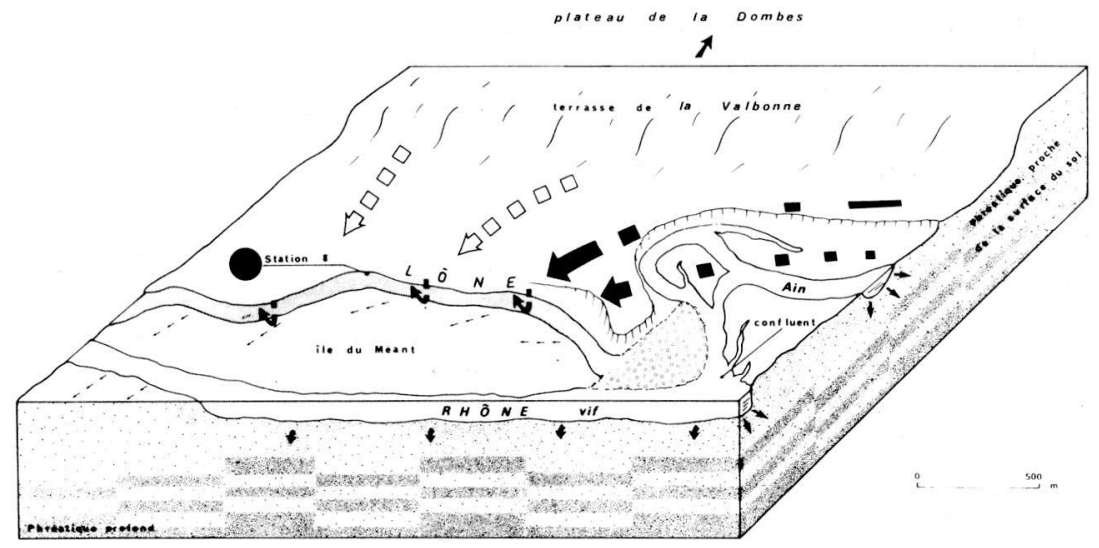

Fig. 2. Courants souterrains dans la zone d'alimentation de la Station 8

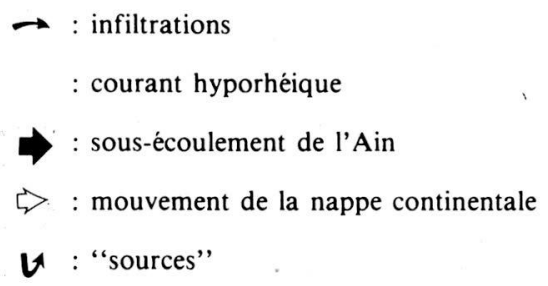

\section{GRANULOMÉTRIE COMPARÉE DES DEUX STATIONS}

Les prélèvements granulométriques effectués au début de la campagne (1975) sont analysés Figure 3. Les particules assez fines (sablons), dont l'influence sur la répartition de la méïofaune ne devrait pas être négligeable, sont mieux représentées en station 8 (pic à $0,18 \mathrm{~mm}$.) et traduisent un débit moyen plus faible.

\section{ANALYSE PHYSICO-CHIMIQUE DES EAUX}

\section{A) Mode de Prélèvement et Méthode d'Analyse}

Après chaque prise de faune, un échantillon d'eau est prélevé, à $60 \mathrm{~cm}$. environ dans le sédiment, à travers la sonde crépinée de type Bou-Rouch restée en 
place (Bou et Rouch, 1967; Bou, 1974). L'eau interstitielle est prise, lentement, au moyen d'une pompe péristaltique adaptée sur un tube "Rilsan" (pompe Wab); ce système est utilisé en écologie phréatique depuis Husmann (1974). Les flacons à double bouchage sont placés en bac isotherme et ramenés au Laboratoire. Quelques mesures sont effectuées in situ: Température, $\mathrm{pH}$, Oxygène. Les autres sont faites au Laboratoire selon les méthodes d'analyse suivantes: lin.

- Oxydabilité au permanganate: détermination à chaud en milieu alca-

- Titre alcalimétrique complet: titrimétrie par $\mathrm{HCl}$ en présence d'indicateur mixte.

- Calcium et Magnésium: dosage simultané par complexométrie à l'E.D.T.A. sur un même échantillon (Rodier, 1975).

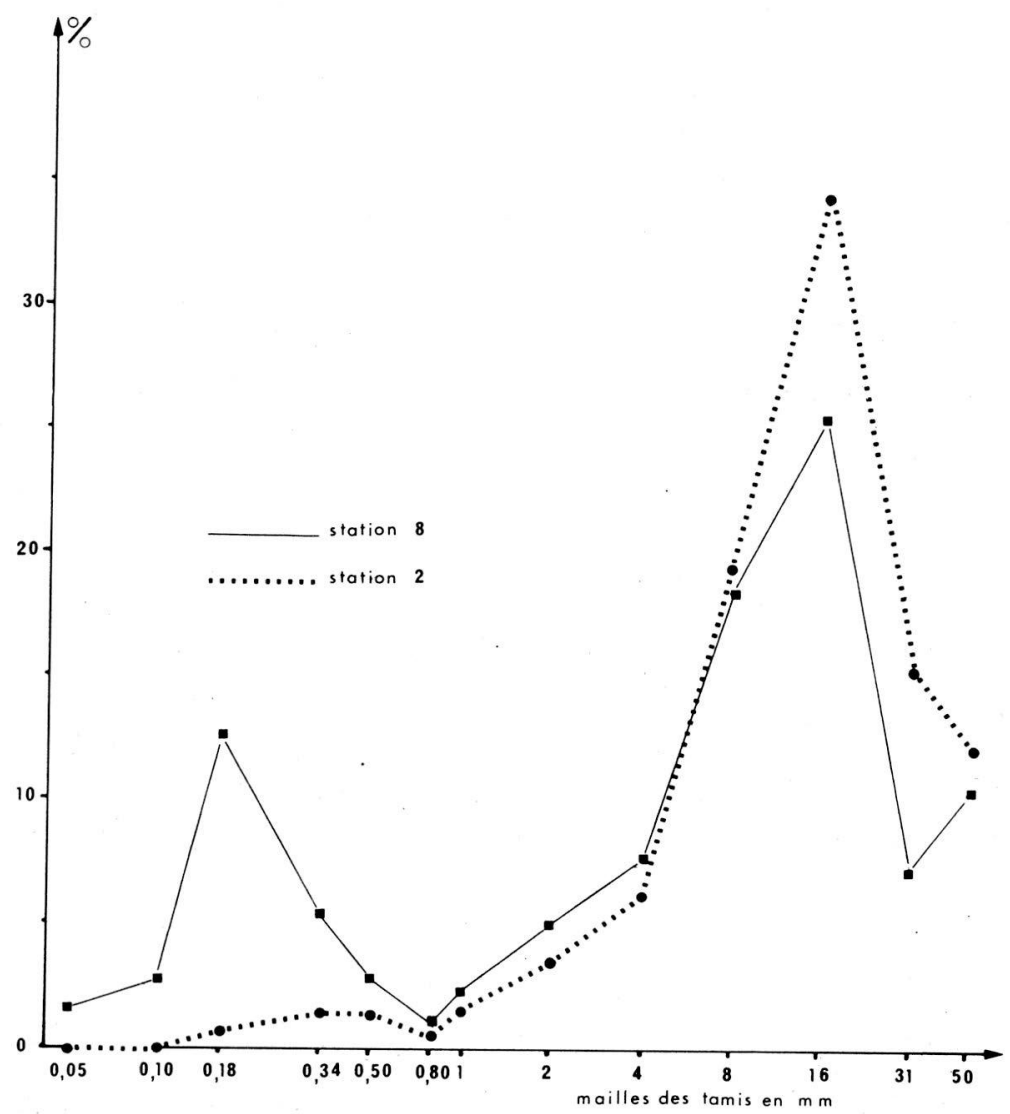

Fig. 3. Granulométrie comparée des stations 2 et 8 . 
- Chlorures: titrimétrie au nitrate mercurique avec indicateur (Diphénylcarbazone).

- Sulfates: dosage par turbidimétrie (Rodier, 1975).

- Nitrates: spectrophotométrie au salicylate de Soude (Rodier, 1975). sver III.

- Orthophosphates: photocolorimètre Hach IR-DR; méthode au Pho-

- Silice dissoute: photocolorimètre Hach IR-DR; méthode aminoacide.

- Fer total: photocolorimètre Hach IR-DR; méthode à la ferrozine.

\section{B) Résultats}

En régime moyen, les stations ne manifestent pas de différences flagrantes:

- le pH est toujours voisin de la neutralité;

- les écarts de température observés sur un cycle annuel ne dépassent pas $6^{\circ} \mathrm{C}$; notons cependant qu'ils sont plus marqués en station 2, car les températures basses, décalées, mais induites par celles du Rhône vif, y sont nettement inférieures à celles de la station 8 :

- l'oxydabilité, en moyenne faible (entre 0 et 1 ), est légèrement supérieure en station 2 ;

- les eaux sont toutes deux très bicarbonatées-calciques $\left(\mathrm{HCO}_{3}^{-}\right.$toujours supérieur à $300 \mathrm{mg} / \mathrm{L} ; \mathrm{Ca}^{++}$entre 80 et $100 \mathrm{mg} / \mathrm{L}$ );

- la seule très forte disparité s'exprime au niveau des teneurs moyennes en silice dissoute; elles sont beaucoup plus élevées (au moins le double) en station 2.

En définitive, c'est à la faveur de deux épisodes hydrologiques extrêmement différents (1976 et 1977) que nous avons pu constater l'originalité de chacune des stations. En effet:

$1^{\circ}$ ) la pluviosité de 1976 fut si faible qu'elle fit subir à la région une sécheresse exceptionnelle pendant les mois d'été (le débit moyen du Rhône pour l'année tomba à $405 \mathrm{~m}^{3} / \mathrm{s}$ ). En conséquence, le para-écoulement souterrain de la rive gauche subit un net "tarissement"; le barrage de Jons maintenant artificiellement un niveau anormalement haut pour un tel débit, les eaux superficielles refluèrent jusqu'en amont de la lône où elles stagnèrent plus de trois mois, contaminant la zone interstitielle.

Pendant ce temps, aucune modification n'intervenait dans le régime hydrique de la station 8 .

$2^{\circ}$ ) dès l'hiver, les précipitations s'accumulèrent, et le début de l'année 1977 fut le cadre d'une crue extrêmement forte du fleuve (du 6 février à la fin du même mois); le pic de crue se situa le 12 février avec un débit de 2540 $\mathrm{m}^{3} / \mathrm{s}$. Depuis cet instant, le Rhône occupa la totalité de son lit majeur, retrouvant ses trajets du début du siècle. Les deux lônes furent donc balayées, pendant trois semaines, par ce flux chargé de limons. La pluviosité se maintint très forte, même après le retour du fleuve dans son lit normal; l'été fut si humide que 1977 est actuellement l'une des deux années les plus arrosées du sì̀cle. Les hauteurs d'eau tombées pendant le mois de juillet à la station -Météo de Lyon-Gerland constituent même un record absolu pour la région avec 191,4 mm. Durant le reste de l'année, les nappes furent hautes et le débit 
phréatique important; le Rhône accusa une très forte moyenne générale avec $728 \mathrm{~m}^{3} / \mathrm{s}$ (débit annuel moyen 1920-1976: $584 \mathrm{~m}^{3} / \mathrm{s}$ ).

Pendant ces deux périodes, l'évolution de la plupart des éléments majeurs de l'eau a été suivie; nous avons extrait de nos résultats six courbes qui résument les deux types de "comportement hydrologique" des stations en ces circonstances (Fig. 4).

On peut y faire les constatations suivantes:

- Température: oscillant habituellement autour d'une moyenne de $11,5^{\circ} \mathrm{C}\left( \pm 3^{\circ}\right)$, la température de la station 2 a subi une très forte hausse pendant la sécheresse de 1976 , avec un pic en juillet à $24,8^{\circ} \mathrm{C}$. Ce pic est dû à la stagnation des eaux dans le sédiment. Corrélativement, la station 8 reste très tamponnée par la nappe continentale et ne dépasse pas un maximum de $14^{\circ} \mathrm{C}$.

Après la crue de 1977 , le fort débit interstitiel ramène les températures dans leur "fourchette" habituelle, la station 8 restant toujours plus chaude en hiver.

- Conductivité électrique: c'est une variable facile à mesurer et qui traduit bien la minéralisation globale de l'eau. Elle met ici en évidence la chute brutale, pendant les trois mois de sécheresse, de la plupart des éléments caractéristiques de l'eau interstitielle en station 2. Après la reprise d'un certain débit souterrain (dès novembre 1976), le milieu récupère très vite sa minéralisation moyenne.

Notons qu'après la forte pluviosité du début de l'année et la crue de février 1977, la station 8 qui n'avait pas semblé notablement affectée par la sécheresse précédente, subit une nette hausse de conductivité dont le sousécoulement de l'Ain, vu la promptitude de la réaction, est sans doute le responsable.

- Sulfates - Calcium: nous avons choisi ces deux ions antagonistes car ils sont tous deux les indicateurs d'un certain type d'eau. En effet, le Rhône superficiel est très chargé en sulfates, drainant dans son cours supérieur (Bassin du Léman) des bancs de Gypse triasique (Juget, et al., 1976); les valeurs sont constamment voisines de $40 \mathrm{mg} / \mathrm{L}$. Nous avons déjà signalé que pendant la sécheresse, le barrage, créant un "faux niveau", fit refluer les eaux jusqu'en amont de la Lône des Pêcheurs. Le brusque pic de sulfates en station 2, et, corrélativement, la retombée extrêmement rapide du Calcium caractérisant l'eau interstitielle, sont la preuve de la contamination du milieu phréatique par les eaux du fleuve... Dès novembre 1976, le "lavage" que produisit la reprise du para-écoulement rendit très vite à la station 2 ses faibles valeurs en sulfates (peu différentes de celles qu'on observe en station 8 ) et ses très fortes concentrations en Calcium $(111,6 \mathrm{mg} / \mathrm{L}$ à cette date).

Là encore, la station 8 resta d'une totale stabilité pendant l'épisode sec. Cependant, après une courte baisse du Calcium immédiatement après la crue (contamination superficielle constatée également en station 2), elle accusa un ressaut très important qui lui fit rejoindre les valeurs élevées de la station 2 . Ceci est sans aucun doute le fait du sous-écoulement de l'Ain, descendant du karst lessivé par les pluies, et participe pour sa plus grande partie à l'augmentation de la conductivité électrique citée précédemment. 

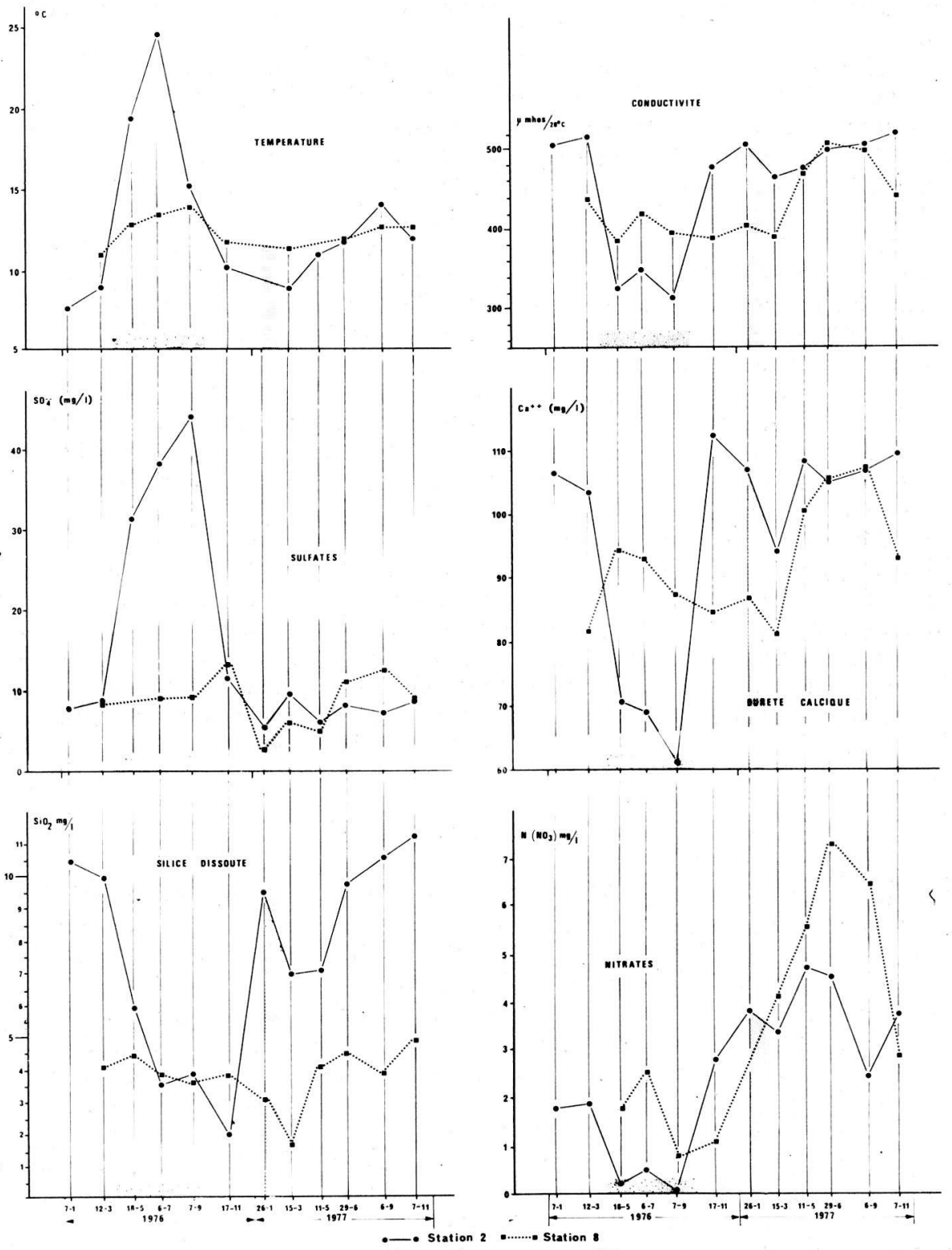

Fig. 4. Évolution de quelques éléments majeurs de l'eau des stations 2 et 8 au cours des épisodes hydrologiques très différents de 1976 et 1977. 
- Silice et nitrates: ces deux derniers éléments manifestent encore les bouleversements qui se sont opérés pendant deux ans dans l'interstitiel régional:

En station 2, la teneus en silice est environ deux fois supérieure à celle de la station 8; elle a même pu être utilisée, avec le Calcium, comme indicateur du para-écoulement de la rive gauche par comparaison avec l'eau du Rhône vif. Elle présente habituellement dans la Lône des Pêcheurs un gradient amont/aval décroissant dû à l'action progressive de la végétation aquatique (Juget, et al., 1976). Pendant les mois secs, ce gradient a totalement disparu, car les valeurs tombent brusquement au niveau de celles de la station 8 , trahissant à nouveau l'invasion de ce secteur par les eaux du Rhône. Encore une fois, dès la reprise du para-écoulement, les taux remontent en flèche. La courte contamination superficielle déjà observée pour le Calcium se manifeste également après la crue de 1977.

- Les nitrates sont en général voisins dans les deux stations; les concentrations en sont proches de celles observées en Angleterre par Gledhill (1977) dans cette frange supérieure du milieu interstitiel. Elles baissent fortement en station 2 pendant la sécheresse, l'eau du Rhône pauvre en nitrates accupant le sédiment.

Mais le plus important phénomène observé est l'augmentation très forte des taux en station 8 pendant le régime phréatique fort, qui participe également à la hausse de la conductivité électrique de l'eau. Ceci représente, compte tenu d'un temps de latence prévisible (le pic est en juillet), la réponse de la nappe aux pluies du début de l'année. En effet l'augmentation du débit général agit sur l'interstitiel profond dont on sait (Agence de Bassin, 1974; S.R.A.E., 1977) que les teneurs en nitrates sont très élevées dans cette plaine de "Miribel-La Valbonne"; elles avoisinent $30 \mathrm{mg} / \mathrm{L}$ à $-15 \mathrm{~m}$.

\section{COMMENTAIRES}

Ce qui précède démontre l'importance de l'analyse physico-chimique en écologie phréatique; ce n'est que trois ans après le début de notre travail sur ces deux stations que nous commençons à bien connaître leur régime hydrologique et les conditions de milieu qu'elles présentent.

Nous avons pu constater la très grande variabilité des composantes de la station 2, qu'elles soient hydrauliques ou physico-chimiques. Au contraire, la station 8 parait extrêmement tamponnée par la présence de la nappe régionale, jusqu'à un certain seuil où le sous-écoulement de l'Ain peut manifester son originalité $\left(\mathrm{Ca}^{++}, \mathrm{Fig}\right.$. 3). Il semble que le vaste réservoir de la plaine de Miribel-La Valbonne, à circulation lente et régulière (débit: $400 \mathrm{1} / \mathrm{sec}$. pour une vitesse moyenne de 3,5 mètres par jour) représente un élément de stabilité incontestable pour les biocénoses interstitielles résidant dans cette station; par opposition, le régime de la station 2, étroitement tributaire du débit du Rhône vif et compliqué par la présence du barrage de JONS, semblerait ne convenir qu'à des organismes supportant des conditions instables.

Ces deux biotopes peuvent cependant, pour des raisons différentes (cf. 
plus haut), constituer chacun une zone privilégiée d'échanges entre les secteurs hypogé et épigé. L'étude faunistique $(c f$. IX) montrera si leurs facteurs de milieu si opposés ont exercé une influence sur la composition des biocénoses.

\section{RÉSUMÉ}

Deux stations phréatiques alimentant en eau interstitielle d'anciens méandres du Rhône ont été suivies depuis 1975. Leurs modes hydrologiques sont très différents (ils sont schématisés sur deux blocs diagrammes):

- l'une (station 2) est un écoulement souterrain parallèle au cours du fleuve, très lié à ses variations de débit, et circulant dans un substrat à granulométrie assez grossière (paraécoulement);

- l'autre (station 8 ) résulte de la confluence de la nappe continentale provenant du plateau de la Dombes (au Nord-Est) avec le sous-écoulement de l'Ain, affluent du Rhône. Les variations de débit du fleuve n'influent pas sur cet écoulement phréatique.

Une étude physico-chimique a permis, à l'occasion de conditions climatiques exceptionnelles (sécheresse-inondation), de confirmer la grande stabilité des facteurs du milieu en station 8 ; par contre, ceux de la station 2 présentent une variabilité originale pour un biotope interstitiel.

\section{OUVRAGES CITÉS}

AGENCE DE BASSIN Rhône - Méditerranée - Corse. 1974. Protection des nappes alluviales - contre la pollution: Site alluvial de l'Est Lyonnais: Etude générale: 190p, 15 cartes.

BOU, C. 1974. Recherches sur les eaux souterraines interstitielles. Ann. Spéléol. 29, 4:611-619.

BOU, C., et ROUCH R. 1967. Un nouveau champ de recherches sur la faune aquatique souterraine. C.R. Acad. Sci. Paris 265:369-370.

B.R.G.M. 1971a. Note de synthèse résumant les principaux enseignements tirés des observations piézométriques: rapport 71. S.G.N. 129 JAL.

B.R.G.M. 1971b. Interprétation de pompages d'essais effectués danş la plaine de l'Est Lyonnais. Rapport 71, S.G.N. 261 JAL.

B.R.G.M. 1972. Interprétation de pompages d'essais effectués dans la plaine de l'Est Lyonnais. Rapport 72, S.G.N. 113 JAL.

B.R.G.M. 1973. Synthèse des connaissances sur le système aquifère de l'Est Lyonnais. Rapport 73, S.G.N. 199 JAL.

B.R.G.M. 1976. Réflexions sur le système aquifère de l'Est Lyonnais. Rapports 76, S.G.N., 355 JAL. - 76, S.G.N. 447 JAL.

DORGELO, J. 1973. Etude de la végétation dans les anciens lits du Rhône et des moustiques qui lui sont liés, de Lyon au confluent de l'Ain. Dipl. Et. Sup. Univ. Claude Bernard, Lyon.

GIBERT, J., GINET, R., MATHIEU, J., REYGROBELLET, J.L., et SEYED-RE̊IHANI, A., 1977. Structure et fonctionnement des écosystèmes du Haut-Rhône français. IV - le peuplement des eaux phréatiques; premiers résultats. Ann. Limnol. 13, 1:83-97.

GLEDHILL, T. 1977. Numerical fluctuations of four species of subterranean amphipods during a five year period. Crustaceana Suppl. 4:144-152.

HUSMANN, S. 1974/75. Versuche zur Erfassung der vertikalen Verteilung von Organismen und chemischen Substanzen im Grundwasser von Talauen und Terrassen; Methoden und erste Befunde. Int. J. Speleol. 6:271-302.

JUGET, J., AMOROS, C., GAMULIN, D., REYGROBELLET, J.L., RICHARDOT M., RICHOUX, Ph., et ROUX C. 1976. Structure et fonctionnement des écosystèmes du Haut-Rhône français. II - Étude hydrologique de quelques bras morts. Premiers résultats. Bull. Ecol. 7, 4:479-492.

JUGET, J., YI, B.J., ROUX, C., RICHOUX, Ph., RICHARDOT-COULET, M., REYGROBELLET, J.L., et AMOROS C. 1979. Structure et fonctionnement des écosystèmes du Haut-Rhône français. VII - Le complexe hydrographique de la Lône des Pêcheurs (ancien méandre du Rhône). Rev. Suisse Hydrol. 41/2:395-417. 
ORTSCHEIT, A. 1975. Le complexe hydrographique du confluent Ill - Rhin en aval de Strasbourg, ses originalités hydrobiologiques. Thèse, 888. U.E.R. Sc. Pharma. Strasbourg.

PINCHAUT, R. 1975. Protection des nappes alluviales: orientations et conclusions des études du site alluvial de l'est lyonnais. Bull. Inform. Comité et Agence de Bassin RhôneMéditerranée-Corse, 9: 34-50.

RODIER, J. 1975. L'Analyse de l'eau. Eaux naturelles, eaux résiduaires, eaux de mer. Dunod ed., Paris, 628 p.

ROUX, A.L. 1976. Structure et fonctionnement des écosystèmes du Haut-Rhône français. I Présentation de l'étude. Bull. Ecol. 7, 4:475-478.

S.R.A.E. RHÖNE-ALPES. 1977. A.S.A. d'irrigation de l'Ain - Casier de Balan: Reconnaissance hydrogéologique. Ministère Agriculture France (rapport interne). 